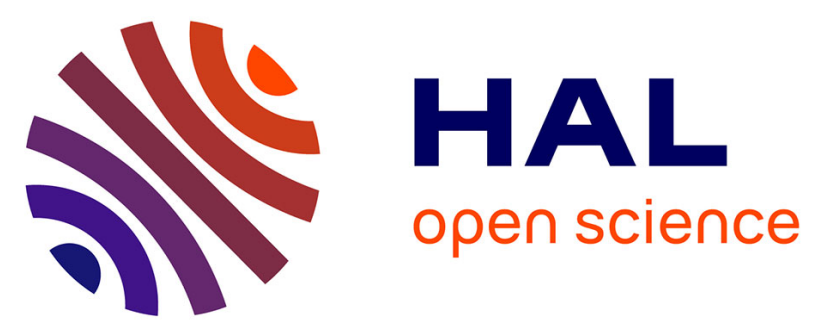

\title{
Interpretations of the real time scattering experiment for polystyrene melts during stress relaxation
}

\author{
François Boué, M. Nierlich, G. Jannink, R.C. Ball
}

\section{To cite this version:}

François Boué, M. Nierlich, G. Jannink, R.C. Ball. Interpretations of the real time scattering experiment for polystyrene melts during stress relaxation. Journal de Physique Lettres, 1982, 43 (16), pp.593-600. 10.1051/jphyslet:019820043016059300 . jpa-00232096

\section{HAL Id: jpa-00232096 https://hal.science/jpa-00232096}

Submitted on 1 Jan 1982

HAL is a multi-disciplinary open access archive for the deposit and dissemination of scientific research documents, whether they are published or not. The documents may come from teaching and research institutions in France or abroad, or from public or private research centers.
L'archive ouverte pluridisciplinaire HAL, est destinée au dépôt et à la diffusion de documents scientifiques de niveau recherche, publiés ou non, émanant des établissements d'enseignement et de recherche français ou étrangers, des laboratoires publics ou privés. 
Classification

Physics Abstracts

$05.40-46.30 \mathrm{~J}-61.12-61.40 \mathrm{~K}-05.90$

\title{
Interpretations of the real time scattering experiment for polystyrene melts during stress relaxation
}

\author{
F. Boué, M. Nierlich, G. Jannink and R. C. Ball \\ Laboratoire Léon Brillouin, C.E.N. Saclay, 91191 Gif sur Yvette Cedex, France
}

(Reçu le 11 février 1982, révisé le 15 juin, accepté le 21 juin 1982)

\begin{abstract}
Résumé. - Nous discutons des observations du facteur de forme statique $S_{t_{\mathrm{R}}}(q)$ d'une chaîne dans un échantillon de polystyrène amorphe $\left(M_{\mathrm{w}}=650000\right)$, mesuré dans le domaine intermédiaire, pour différentes durées de relaxation $t_{\mathrm{R}}$ après une déformation uniaxiale. Les valeurs du facteur de forme tirées de cette expérience sont reproduites dans la lettre précédente à ce Journal. Elles sont représentées ici de deux manières différentes. La première découle d'une loi de superposition $q t_{R}^{1 / 4}$ pour des durées comprises entre le temps de Rouse et le temps terminal de relaxation de la contrainte [1]. La seconde découle de l'existence supposée, à grand $q$, d'un temps de relaxation indépendant de $q$, c'est-à-dire de l'échelle d'observation, et égal au temps terminal.
\end{abstract}

\begin{abstract}
We discuss observations of the static form factor $S_{t_{\mathrm{R}}}(q)$ of the single chain, in amorphous polystyrene $\left(M_{\mathrm{w}}=650000\right)$ in the submolecular range for different durations of relaxation $t_{\mathrm{R}}$ after a sudden uniaxial elongation. The data resulting from these observations are given in the preceding letter to this Journal. They are displayed here in two different representations. The first is derived from a $q t_{\mathrm{R}}^{1 / 4}$ superposition law for durations $t_{\mathrm{R}}$ between the Rouse time and the terminal stress relaxation time, as in reference [1]. The second representation is derived from the assumed existence of a $q$ independent relaxation time, equal to a macroscopic quantity, the terminal relaxation time.
\end{abstract}

The dynamics of polymer melts far from equilibrium is today a subject of great interest. Predictions [2] are made for instance concerning the stress relaxation at constant strain after an initial uniaxial deformation. However, we find few explicit results concerning the relaxation process of the coil configuration towards equilibrium. A quantity of interest, which is observable in scattering experiments, is the static form factor. Only approximate formulations for the time dependence of this quantity are given. The approximations consist either in ignoring effects of coil entanglements [3] or in accounting for these effects by using a simple idea [4, 5] based on the reptation model.

Neutron scattering data are now available for the time dependence of the static coil form factors $S_{t_{\mathbf{R}}}(\mathbf{q})$ for polystyrene in the melt state during stress relaxation, at constant macroscopic strain, after an initial uniaxial deformation by a factor 3. In the preceding letter of this Journal [6], a table is displayed in which these static form factors are given as a function of the scattering vector $\mathbf{q}$ in the submolecular range

$$
7 \times 10^{-3}, 2 \times 10^{-1} \AA^{-1}
$$


successively in the directions parallel and perpendicular to the deformation direction, for several values of the duration of relaxation $t_{\mathrm{R}}$ after the initial deformation, and for several stretching temperatures $\mathbb{T}$ (above the glass temperature). The parameters $t_{\mathrm{R}}$ and $\mathbb{T}$ are adjusted in such a way that

$$
T_{\text {Rouse }} \leqslant t_{\mathrm{R}} \leqslant T_{\text {rep }}
$$

where $T_{\text {Rouse }}$ and $T_{\text {rep }}$ are the so-called first Rouse and reptation relaxation times [2, 7].

We wish now to interpret the scattering data, using available theoretical expressions. We know that these expressions are not adequate, because they are based on incorrect or crude assumptions. Confrontations reveal however several facts of interest which are discussed in this letter. In order to do so, we use successively two representations of the data suggested by these theories.

1. The $q t^{1 / 4}$ superposition representation. - We first test a time dependence of the static form factor $S_{t_{R}}(\mathbf{q})$ which is typically derived from the Rouse equation of motion. In a paper published several years ago, Daoudi calculated the time and reciprocal space dependence of $S_{t_{R}}(q)$ for a single chain returning to equilibrium after an affine deformation. His prediction is, in the asymptotic range

$$
S_{t_{\mathbf{R}}}(\mathbf{q})=S_{\infty}(\mathbf{q}) \times f\left(q / q^{*}\right)
$$

where

$$
q^{*} \propto \frac{1}{R_{\mathrm{giso}_{\mathrm{s}}}}\left(t_{\mathrm{R}} / T_{\text {rep }}\right)^{-1 / 4} \quad\left(\propto N^{3 / 4-1 / 2}\right)
$$

and $R_{\mathrm{g}_{\text {iso }}}$ is the radius of gyration of a Brownian chain of $N$ monomers. The scaling of $S_{t_{\mathrm{R}}}(\mathbf{q}) / S_{\infty}(\mathbf{q})$ with the product $q t_{\mathrm{R}}^{1 / 4}$ is a characteristic result of the Rouse equation. This equation describes the motion of a free coil. However, a $q t^{1 / 4}$ superposition could be obtained from the description of the motion of a coil constrained in a fixed tube (even if its diameter $D$ tends to zero); in particular, this is the case for the reptation model for small values of $t / T_{\text {rep }}$.

The equilibrium form factor $S_{\infty}(\mathbf{q})$ is assumed to be the same as the isotropic form factor; we can thus write, in the asymptotic limit $\left(q R_{\mathrm{g}} \geqslant 4\right)$,

$$
S_{\infty}(\mathbf{q}) \propto 1 /\left(q^{2}+\frac{1}{R_{\mathrm{g}}^{2}}\right)
$$

which gives another formulation of equation (2)

$$
q_{\|}^{2}\left(\frac{1}{S_{t_{\mathbf{R}}}}\left(q_{\|}\right)-\frac{1}{S_{t_{\mathbf{R}}}}(0)\right)^{-1}=f\left(q t_{\mathrm{R}}^{1 / 4}\right) .
$$

In reference [1] we have verified the predictions expressed in (4) for all the samples stretched at $128^{\circ} \mathrm{C}$ and $134^{\circ} \mathrm{C}$, in the lower part of the asymptotic range. We now have data in three parts of the asymptotic range. These data are first normalized in such a way that they are joined together continuously. We can check the behaviour (4) in the whole asymptotic range. From $S_{t_{\mathbf{R}}}(q)$ values, the quantity expressed in the left term of (4) is plotted versus $\log _{10} q+\frac{1}{4} \log _{10} t_{\mathrm{R}}$.

Consider now samples stretched at a same temperature ; if equation (4) is valid, plots for different values of $t_{\mathrm{R}}$ should coincide. Figure $1 a$ shows a plot for two samples stretched at $128^{\circ} \mathrm{C}$, where $t_{\mathrm{R}}=8 \mathrm{~min}$. for one, and $30 \mathrm{~min}$. for the other. The coincidence appears to be good; however we should account for the increasing uncertainty of our data as $q$ increases. The same qualification applies to figure $1 \mathrm{~b}$, which shows the behaviour of data from two samples stretched at $134^{\circ} \mathrm{C}$. 
(a)

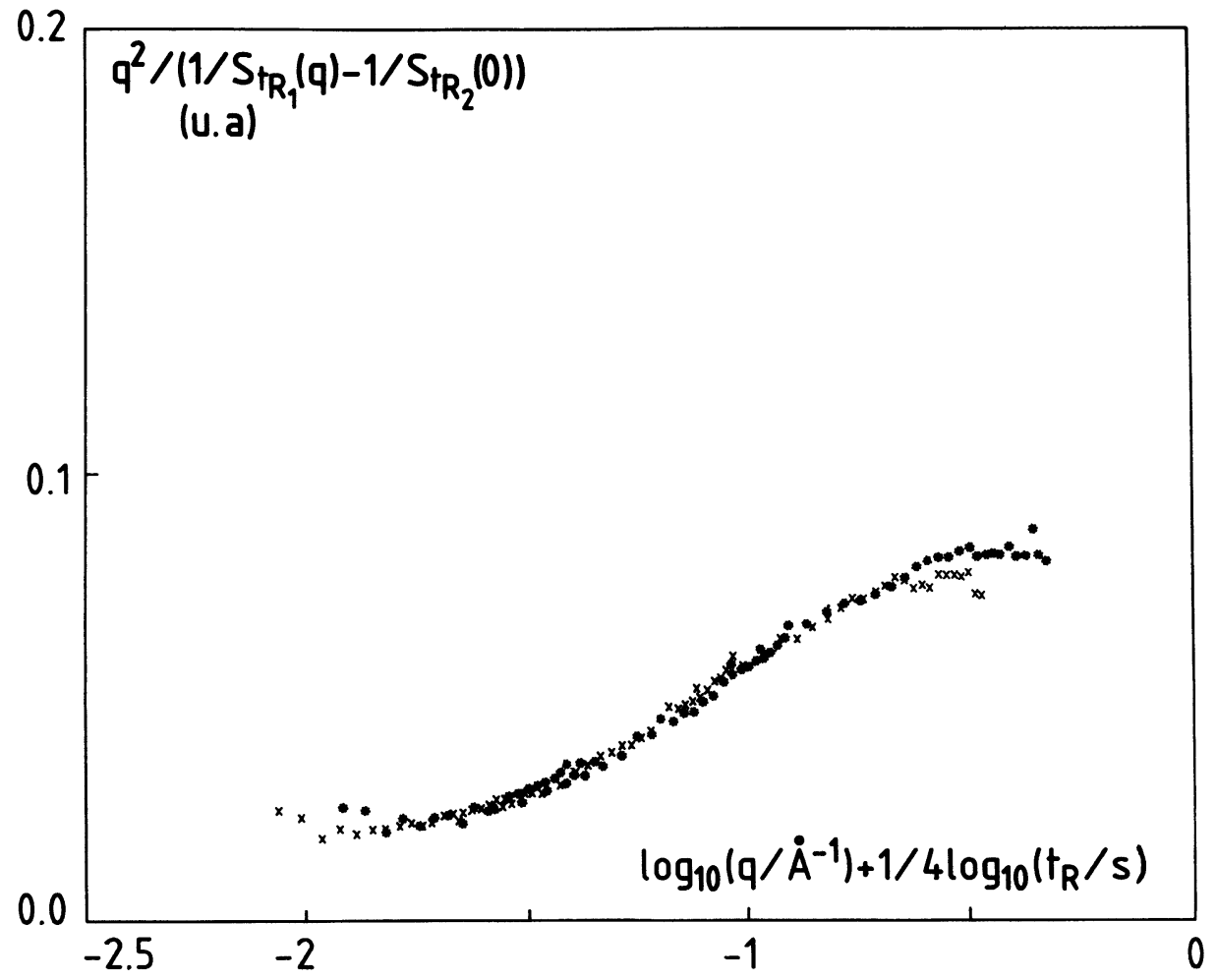

\section{$0 . 2 \longdiv { q ^ { 2 } / ( 1 / S _ { t _ { R _ { 1 } } } ( q ) - 1 / S _ { t _ { R _ { 2 } } } ( 0 ) ) }$} (U.a)

(b)

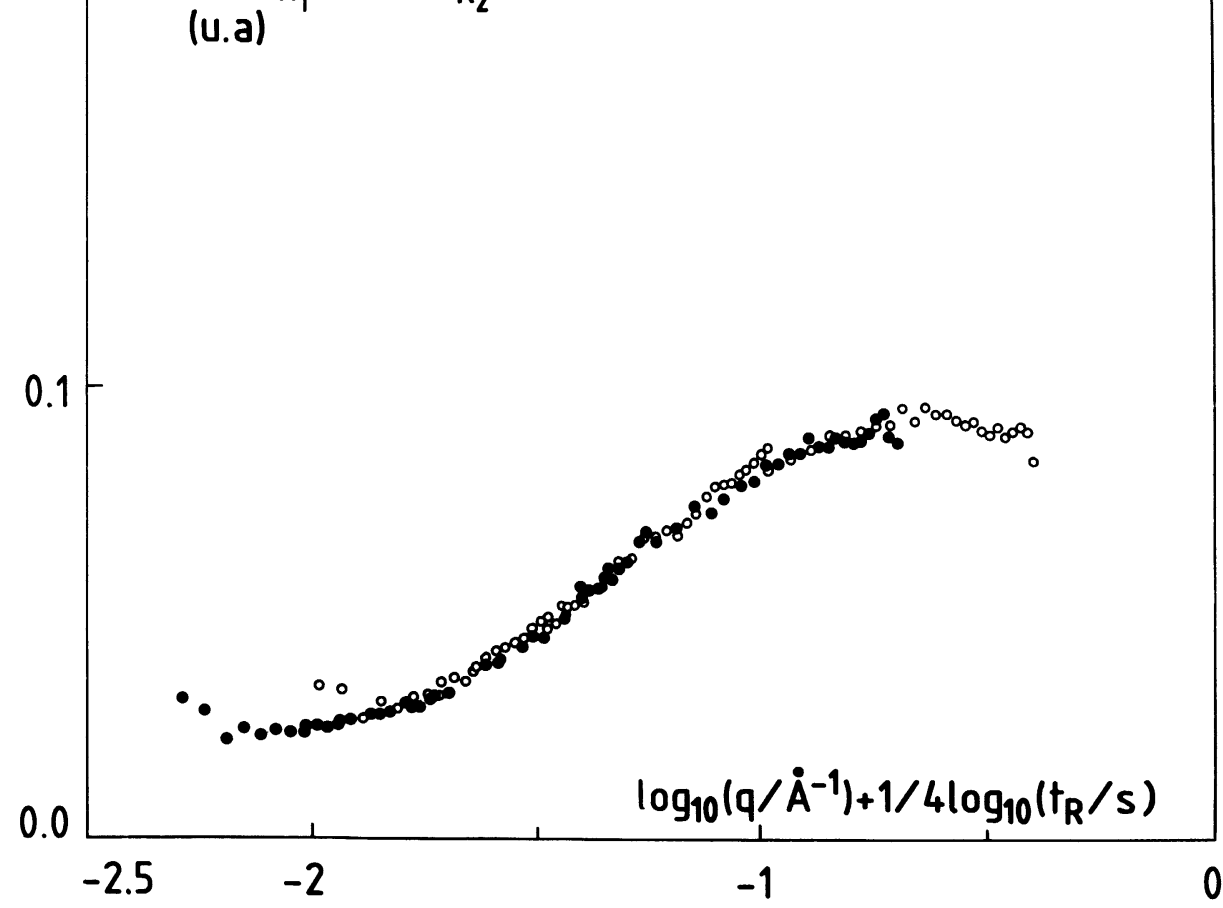

Fig. 1. - Plots of the quantities $q^{2}\left[\frac{1}{S_{t_{\mathrm{R}}}\left(q_{\|}\right)}-\frac{1}{S_{t_{\mathrm{R}_{\mathrm{i}}}}(0)}\right]$ versus $\log _{10} q+\frac{1}{4} \log _{10} t_{\mathrm{R}_{\mathrm{i}}}$ where $S_{t_{\mathrm{R}}}(q)$ is the coil form factor at time $t_{\mathrm{R}}$ and where the index i simply refers to the sample. Each figure concerns a couple of samples. a) $T=128{ }^{\circ} \mathrm{C}: \times$ sample $43, t_{\mathrm{R}_{43}}=8 \mathrm{~min}$; * sample $46, t_{\mathrm{R}_{46}}=30 \mathrm{~min}$. b) $T=134{ }^{\circ} \mathrm{C}$ : - sample $49, t_{\mathrm{R}_{49}}=1$ min.; O sample $50, t_{\mathrm{R}_{50}}=16 \mathrm{~min}$. 
For samples 44 and 43 , the superposition of the two form factors is not as good as in the preceding cases. However, within the error bars, it can still be considered as valid.

2. The factorization representation. - We now present the other possible time dependence for $S_{t_{\mathrm{R}}}(\mathbf{q})$ announced in the introduction. Its starting point is here, clearly, the existence of a time dependent " tube » in which the observed coil is constrained to move. The model is based on the manner in which this tube renews its configuration. Consider the samples just after the step strain, when the coils are assumed to be deformed affinely with respect to the sample. The tube associated with such a coil will be called the affine or " old » tube. After this first instant, the chain progressively disengages by its two ends from the old tube, creating a new isotropic tube in replacement.

The reasoning is clearly applied in reference [8], in the calculation of the dynamic structure factor $S(q, t)$ in naturally fluctuating isotropic polymer melts. We now apply the same reasoning assuming that the values of $q$ are high enough compared to the inverse sizes of both new and old parts of the tube. In this case, contributions from old and new parts to the time dependence of the form factor are additive. Moreover the contribution of the old part at given time $t_{\mathrm{R}}$ is simply proportional to the number $N_{\text {old }}\left(t_{\mathrm{R}}\right)$ of units pertaining to that part. In this case we have :

$$
N S_{t_{\mathbf{R}}}(\mathbf{q})=N_{\text {new }}\left(t_{\mathbf{R}}\right) S_{\infty}(\mathbf{q})+N_{\text {old }}\left(t_{\mathbf{R}}\right) S_{\text {old }}(\mathbf{q})
$$

with

$$
N_{\text {new }}\left(t_{\mathrm{R}}\right)+N_{\text {old }}\left(t_{\mathrm{R}}\right)=N
$$

which implies

$$
S_{\infty}(\mathbf{q})-S_{t_{\mathbf{R}}}(\mathbf{q})=\frac{N_{\text {old }}\left(t_{\mathrm{R}}\right)}{N}\left[S_{\infty}(\mathbf{q})-S_{\text {old }}(\mathbf{q})\right]
$$

If we assume, following [2], [6] that

$$
\begin{aligned}
N_{\text {old }}\left(t_{\mathrm{R}}\right) & =N \sum_{N, \text { odd }=1} \frac{1}{p^{4}} \exp -\frac{p^{2} t}{T_{\text {rep }}} \\
& \sim N \mathrm{e}^{-t / T_{\text {rep }}} \text { for } t / T_{\text {rep }} \gtrsim 1
\end{aligned}
$$

we obtain a simple factorized structure

$$
\left[S_{\infty}-S_{t_{R}}\right](\mathbf{q})=\mathrm{e}^{-t / T_{\text {rep }}} g(\mathbf{q})
$$

analogous to that used for $S(q, t)[6]$.

A good test for equation (8) would be obtained from the plot of

$$
B\left(q_{\|}, t_{\mathrm{R}}\right)=\ln \left[S_{\infty}\left(q_{\|}\right)-S_{t_{\mathbf{R}}}\left(q_{\|}\right)\right]
$$

versus $t_{\mathrm{R}}$; namely the time $t_{\mathrm{R}}$ dependence in this representation is linear, with a slope $1 / T_{\text {rep }}$, and this slope is independent of $q$. We have plotted the mean slope for two values of $t_{\mathrm{R}}$,

$$
\frac{B\left(q_{\|}, t_{\mathbf{R}_{2}}\right)-B\left(q_{\|}, t_{\mathrm{R}_{1}}\right)}{t_{\mathrm{R}_{2}}-t_{\mathrm{R}_{1}}}
$$

versus $q$, and we check whether this quantity is independent of $q$, or not. Figure $2 a$ shows this quantity for the same couple of samples as in figure $1 a\left(T=138^{\circ} \mathrm{C}, t_{\mathrm{R}_{1}}=8 \mathrm{~min}\right.$., $t_{\mathrm{R}_{2}}=30 \mathrm{~min}$. $)$. Figure $2 b$ corresponds to figure $1 b\left(T=134{ }^{\circ} \mathrm{C}, t_{\mathrm{R}_{1}}=1 \mathrm{~min}\right.$.; $t_{\underline{R}_{2}}=16 \mathrm{~min}$.). In the two figures, 

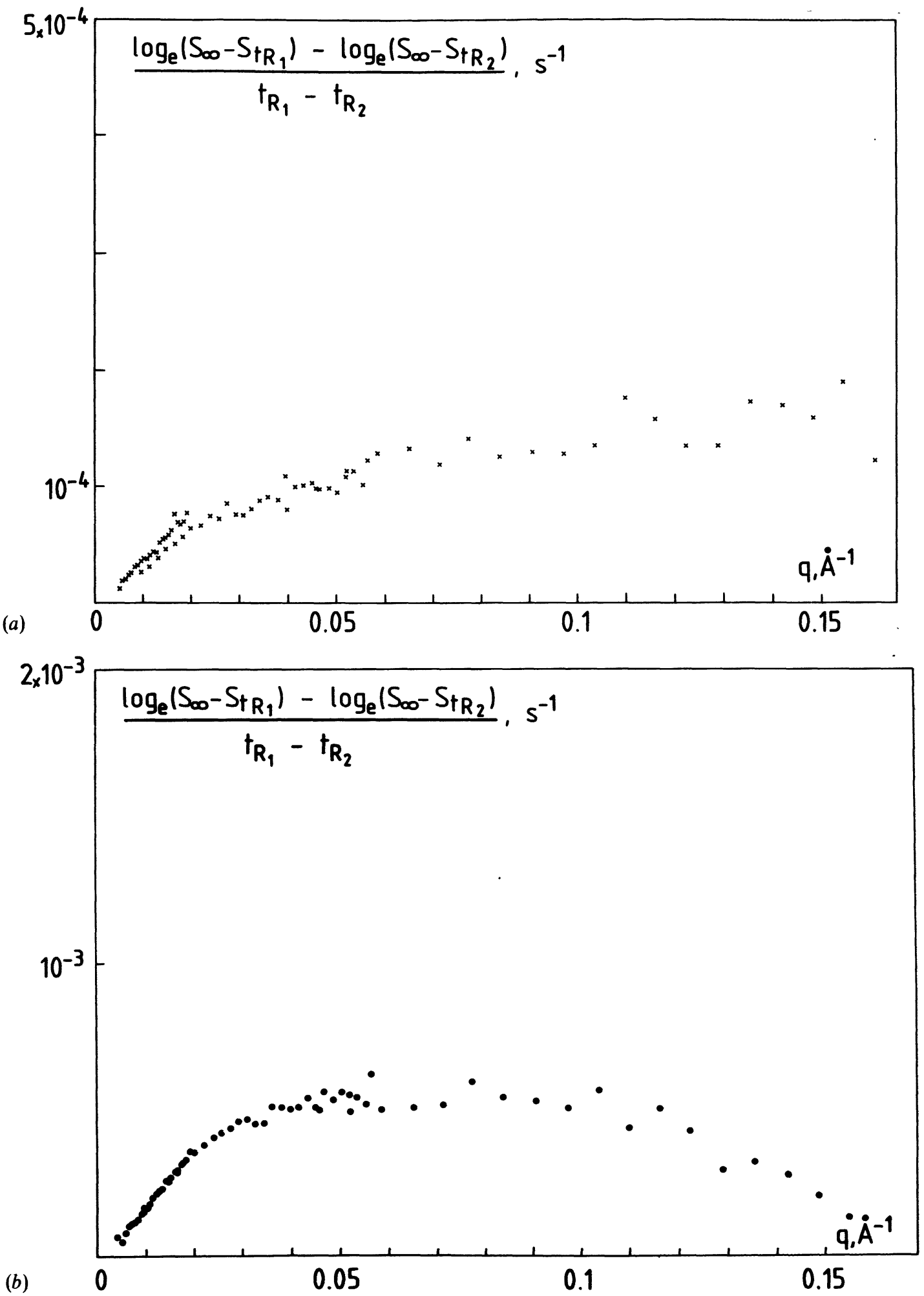

Fig. 2. - Plots of the quantities

$$
\ln \left(-S_{t_{\mathbf{R}_{\mathbf{i}}}}\left(q_{\|}\right)+S_{\infty}(q)\right)-\ln \left(-S_{t_{\mathbf{R}}}\left(q_{\|}\right)+S_{\infty}(q)\right) /\left(t_{\mathbf{R}_{\mathbf{i}}}-t_{\mathbf{R}} .\right)
$$

for two couples of samples (i,j). The samples are the same as in the preceding figure. $a)$ $\mathrm{i}$ : sample $43, t_{\mathrm{R}_{43}}=8 \mathrm{~min}$; j : sample $46, t_{\mathrm{R}_{46}}=30 \mathrm{~min}$. b) $T=134{ }^{\circ} \mathrm{C}: \mathrm{i}:$ sample $49, t_{\mathrm{R}_{49}}=1 \mathrm{~min}$; $\mathrm{j}:$ sample $50, t_{\mathrm{R}_{50}}=16 \mathrm{~min}$. 
$2 a$ and $2 b$, this quantity appears to increase at low $q_{\|}$values and seems to reach a plateau. The existence of such a plateau is predicted by the factorization. However a more cautious discussion is needed at this stage.

3. Discussion. - We have now plotted the same neutron scattering data in the two different representations (4) and (10). These representations are not compatible when applied to a same time and reciprocal space interval. In a first $q$ subrange, corresponding to the lowest values, up to $5 \times 10^{-2} \AA^{-1}$ (approximately), the representation (4) shows that a $q t_{\mathrm{R}}^{1 / 4}$ superposition law (2) is well obeyed; in the same $q$ subrange, the factorization law (8) is not verified. In the second subrange, it is difficult to reject the validity of one or the other law. A more decisive test will be obtained by collecting data for several other relaxation durations $t_{\mathrm{R}}$, because the two laws (2) and (8) could give a plateau in $q$ for representation (10) but law (2) cannot give a $t_{\mathrm{R}}$ independent value of this plateau. For example, developing the function $f(x)$ of equation (2), as expressed in reference [3], for large $x$ we have :

$$
f(x) \simeq 1-\frac{1}{x^{2}}
$$

and

$$
S_{\infty}(\mathbf{q})-S_{t_{\mathrm{R}}}(\mathbf{q})=S_{\infty}(\mathbf{q})\left(\frac{1}{q^{2} R_{\mathrm{g}}^{2}}\left(\frac{T_{\mathrm{rep}}}{t_{\mathrm{R}}}\right)\right)^{1 / 2} .
$$

The slope (10) is then given by the expression

$$
\frac{\mathrm{d}}{\mathrm{d} t} \ln \left(S(q)-S_{t_{\mathbf{R}}}(q)\right)=-\frac{1}{2 t_{\mathrm{R}}}
$$

If the factorization assumption (5) is valid, the resulting value of the plateau should be independent of $t_{\mathrm{R}}$ and equal to $T_{\text {rep }}$, for $t_{\mathrm{R}} / T_{\text {rep }}>1$.

We have not enough data to carry such a test. However two comments can be made in favour of the factorization model.

The first concerns the value $\frac{1}{T_{\text {h.q. }}}$ of the plateau in figure 2 .

$$
\begin{aligned}
& \frac{1}{T_{\text {h.q. }}} \sim 1.3 \times 10^{-4} \mathrm{~s}^{-1} \text { at } T=128^{\circ} \mathrm{C} \\
& \frac{1}{T_{\text {h.q. }}} \sim 5 \times 10^{-5} \mathrm{~s}^{-1} \text { at } T=134{ }^{\circ} \mathrm{C} .
\end{aligned}
$$

We compare this value to two experimental evaluations of $1 / T_{\text {rep }}$. We obtain the first evaluation by plotting, from data of reference [1] for the same samples,

$$
C\left(t_{\mathrm{R}}\right)=\ln \left[1-\frac{R_{\mathrm{g}}^{2}\left(t_{\mathrm{R}}\right)}{R_{\mathbf{g}_{\text {iso }}}^{2}}\right]
$$

versus $t_{\mathrm{R}}$. We obtain straight lines. The reptation models predicted such a linear variation, of slope $1 / T_{\text {rep, }}$ which we thus evaluate from the slopes of $C\left(t_{\mathrm{R}}\right)$ :

$$
\begin{array}{lll}
\frac{1}{T_{\text {r.g. }}} \sim 10^{-4} \pm 0.5 \times 10^{-4} \mathrm{~s}^{-1} \quad \text { at } & T=128^{\circ} \mathrm{C} \\
\frac{1}{T_{\text {r.g. }}} \sim 4 \times 10^{-4} \pm 2 \times 10^{-4} \mathrm{~s}^{-1} \text { at } & T=134^{\circ} \mathrm{C}
\end{array}
$$


The two values, $1 / T_{\text {h.q. }}$, corresponding to relaxation process in the submolecular range, and $1 / T_{\text {r.g. }}$, corresponding to relaxation process in molecular range, are equal.

A second evaluation of $1 / T_{\text {rep }}$ is done in reference [1] from the comparison of our stress relaxation data with the data of Tobolsky and coworkers. We use this evaluation made for $T=117^{\circ} \mathrm{C}$ and use a Williams Landel Ferry superposition [1,3] to obtain, for the temperatures of our experiment

$$
\begin{aligned}
\frac{1}{T_{\text {ter }}} & \sim 0.6 \times 10^{-4} \text { at } 128^{\circ} \mathrm{C} \\
& \sim 2.5 \times 10^{-4} \text { at } 134^{\circ} \mathrm{C} .
\end{aligned}
$$

The values of $1 / T_{\text {h.q. }}, 1 / T_{\text {r.g. }}$ and $1 / T_{\text {ter }}$ are of the same order of magnitude. This is in favour of the factorization model.

Finally we present a last observation concerning the anisotropy in orientation with respect to the elongation axis. Equation (6) can lead us to consider

$$
D_{t_{\mathbf{R}}}(q)=\frac{S_{\infty}\left(q_{\|}\right)-S_{t_{\mathbf{R}}}\left(q_{\|}\right)}{S_{t_{\mathbf{R}}}\left(q_{\perp}\right)-S_{\infty}\left(q_{\perp}\right)}
$$

which is plotted in figure 3 , versus $q$, for different $t_{\mathbf{R}}$.

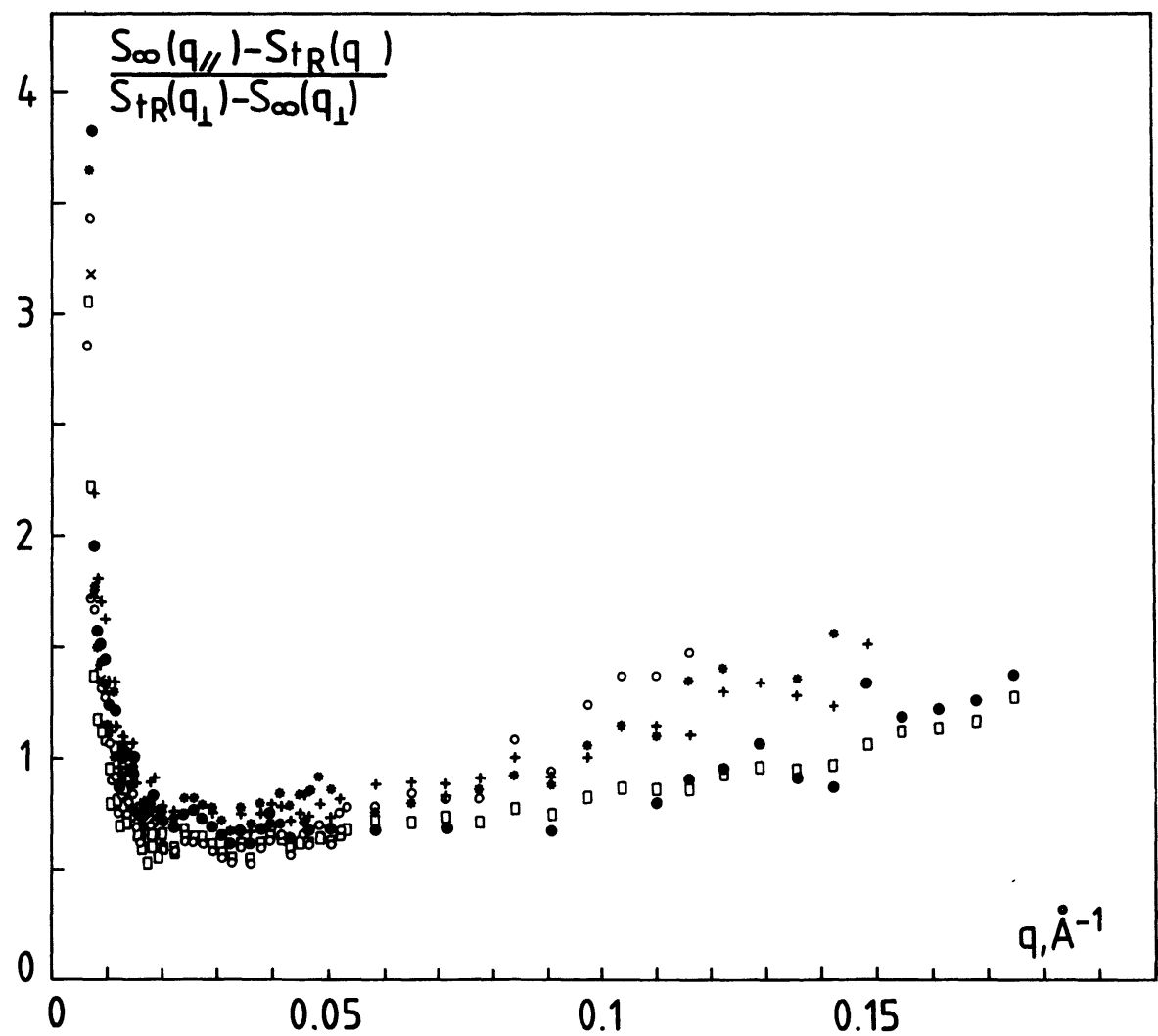

Fig. 3. - Plot of the quantities $\left(S\left(q_{\|}\right)-S_{t_{\mathrm{R}_{\mathrm{i}}}}\left(q_{\|}\right)\right) /\left(S_{t_{\mathrm{R}_{\mathrm{i}}}}\left(q_{\perp}\right)-S\left(q_{\perp}\right)\right)$ versus $q$ for samples $44(+)-T=128^{\circ} \mathrm{C}$, $t_{\mathrm{R}}=60 \mathrm{~s}-, 43(\times), 46(*), 49(\bullet), 50(O)$ - same samples as in figures 1 and 2 - and another sample stretched at lower temperature, $T=113{ }^{\circ} \mathrm{C}$ (sample $71, t_{\mathrm{R}}=10 \mathrm{~s},(\square)$ ). At intermediate $q$, the master curve is a plateau. At high $q$, values of the displayed quantities strongly depend upon the value used to represent the average sample thickness. 
We observe that, all the curves are superposed, up to the highest observed $q$ values. We note that this is observed not only for data previously displayed in this paper, but also for data from samples stretched at lower temperature, corresponding to much lower values of $t_{\mathrm{R}} / T_{\text {rep }}$. The master curve reaches a value independent of $q$, which has been noted to vary with the elongation ratio $\lambda$.

4. Summary. - We have tried to fit two formulas derived from two calculations of $S_{t_{\mathrm{R}}}(q)$ with our experimental data. The first calculation, made by Daoudi, is said to apply in a range of $q$ and $t_{\mathbf{R}}$ which covers the experimental range of the data presented here. While this calculation is said to be derived from a reptation motion of the chain in the tube, it does not seem clear to us whether it takes into account the effect of localization inside the tube. The second calculation, which we are proposing, is valid only for times $t_{\mathrm{R}}$ of the order of the disengagement time and for high values of $q$. The derived formula is not in agreement with the formula of Daoudi.

Comparing the formula to the data, we have to conclude the following :

- the formula given by Daoudi, valid for all $q$ 's, fits satisfactorily the data in the entire $q$ and $t_{\mathrm{R}}$ range measured.

- the asymptotic formula given by us, valid for large $q$ 's, also fits the data in the large $q$ range, with the same accuracy.

Thus we are not able to decide between the two formulas, and two points have to be made clear : the first point, related to the large $q$ range, is the explanation of how the same data can be fitted by two fundamentally different formulas : one explanation has been given above, namely that the number of different times $t_{\mathrm{R}}$ at which the form factor is measured is too small to differentiate the $t_{\mathrm{R}}$ dependence of the observed plateau (10). In that case the problem can be solved by gathering further data at other values of $t_{\mathrm{R}}$. The second point is the relation between the tube model and the Daoudi formula : it is not obvious if this formula applies partially to the reptation process or only to a Rouse relaxation process.

Finally we remark that it is possible that the data discussed here correspond to times $t_{\mathrm{R}}$ which are between two characteristic time ranges. One range would be centred around $T_{\text {Rouse }}$ which is here the time for equilibration of the linear density : within this range it would be possible to represent the motion of the chain by a Rouse-like motion yielding the characteristic time and space superposition discussed above. The second range is centred around $T_{\text {rep }}$, and within this range, a time and space superposition would be valid for large $q$ 's. The fact that the data are measured between these two time ranges could explain our difficulty to adopt or to reject one of these two formulas.

Acknowledgments. - We gratefully acknowledge helpful discussions with P. G. de Gennes. We thank J. des Cloizeaux for his comments on the manuscript.

\section{References}

[1] BouÉ, F., Nierlich, M., Jannink, G., Ball, R. C., J. Physique 43 (1982) 137-148.

[2] Doï, M., Edwards, S. F., J. Chem. Soc. Faraday Trans. 274 (1978) 1789, 1802, 1818.

[3] Daoudi, S., J. Physique 38 (1977) 731.

[4] De Gennes, P. G., private communication.

[5] BaLl, R., BouÉ, F., unpublished.

[6] BouÉ, F., Nierlich, M., JANnink, G., Ball, R., same issue.

[7] De Gennes, P. G., Scaling Concepts in Polymer Physics (Cornell University Press) 1979.

[8] De Gennes, P. G., J. Physique 42 (1981) 735-740. 